\section{Olaosebikan RR Ademola-Popoola DS Yusuf AS Oyinloye OI \\ Ernest SK \\ Olorunsola B \\ Ayeni AS \\ Oladele DM}

DOI:http://dx.doi.org/10.4314/njp.v42i4.12

Accepted: 24th June 2015

Olaosebikan RR ( $\mathbf{\Sigma}$ )

Ernest SK, Olorunsola B, Ayeni AS

Oladele DM

Department of Paediatrics,

Yusuf AS

Department of Surgery

Ademola-Popoola DS

Department of Ophthalmology

Oyinloye OI

Departments Radiology

University of Ilorin Teaching Hospital

Email: laoshrsk@yahoo.com

\title{
Blindness in tuberous sclerosis: A case report
}

\begin{abstract}
Tuberous sclerosis (TS) is inherited as an autosomal dominant trait with variable penetrance characterised by glial cell tumor which arises from the cerebral and the retina. Blindness in association with Tuberous sclerosis (TS) is rare. When visual loss occurs it may be associated with hamartomas from retinal or optic nerve involvement or from intracranial (brain) tumours that affect either the part of the brain that processes visual information or from optic nerve damage following raised intracranial pressure. Very few cases of TS with blindness have been reported globally. Deterioration in academic performance might be the first pointer to the visual impairment.

We report a case of a 13 year old girl who presented with increasing number of facial rash over an 11 years period, recurrent headache and deteriorating academic performance of 1year and loss of vision of 6 months with a recent episode of convulsion. Similar skin rashes without other associated symptoms were noticed on the mother and one of the younger siblings.

She was a Tanner stage one in development. She had facialangiofibromas, shagreen patches over the left hypochondria, back regions and face. Ophthalmic evaluation showed a visual acuity of being able to count fingers at not more than one meter from the face and only perception of light
\end{abstract}

in the right and left eye respectively, both eyes had brisk pupillary activities, good mydriasis and clear media. The retinal and optic nerve head appeared normal in the right eye whereas in the left eye was a huge tuberous hamartoma of the optic disc and macular as well as generalised vascular occlusion and subretinal fluid.

The Computerized tomography (CT) scan showed an Intraventricular tumour, with calcification within the tumours and subependymal. There was associated obstructive hydrocephalus. Patient was managed by a multidisciplinaryteam of ophthalmologists, neurosurgeons and radiologists, coordinated by a paediatrician.

Conclusion: The diagnosis of tuberous sclerosis complex (TSC) was based on the lesions found on clinical examination, imaging, and pathologic studies. The blindness was multi-factorial in cause including intracranial, retinal and optic nerve tumours. Comprehensive medical history, detailed physical examinations and neuroimaging study are essential in making a diagnosis of TSC. Our patient was mis-diagnosed at various health facilities for many years. This delay in making appropriate diagnosis and instituting treatment could have contributed to the eventual outcome.

Keywords: Tuberous sclerosis, blindness, deteriorating academic performance,

\section{Introduction}

Tuberous sclerosis complex (TSC) is an inherited neuro- cutaneous syndrome characterised by development of hamartoma in virtually all organs in the body, commonly in the skin, brain, lung, bones, kidney and the 
eyes ${ }^{1}$. TSC is inherited as an autosomal dominant trait with variable penetrance and a prevalence of 1 in 6,000 to 10,000 individuals $^{2}$. The earlier in life a patient presents with symptoms and signs of tuberous sclerosis the greater is the like hood of mental retardation. TSC is associated with various types of intracranial tumours including cortical tubers, subependymal nodules and glial cell tumours. Intracranial tumours can have different presentations ranging from recurrent headache, blindness or seizure. Comprehensive medical history, detailed physical examinations and neuroimaging study are essential in making a diagnosis of TSC. We present this 13 year old whose TS was not diagnosed until she had significant life threatening deterioration and loss of vision in both eyes.

\section{Case presentation}

A 13 year old girl presented with increasing number of facial rash over an 11year period, recurrent headache and deteriorating academic performance of 1year and loss of vision of 6months. There was a recent episode of convulsion. Similar skin rashes without other associated symptom were noticed on the mother and one of the younger siblings. The facial rashes were initially flat and dark but subsequently became raised, dark and wider in circumference.Other rashes of various sizes were noticed after births on the abdomen, back and thighs, these became coalesced to form a raised roughen edge as the child grew.

The rashes were dark, firm small nodules, $1-5 \mathrm{~mm}$ diameter symmetrically distributed over the malar region.Figure 1. There were shagreen patches over the left hypochondria and back regions. There were no ash leaf spots or areas of lightening on skin. There is a similar rash in mother noticed since childhood and in a 7 month old sibling.

Recurrent frontal, throbbing headache lasting about three hours twice a week, relievable by analgesia was noticed in the preceding year prior to admission. There was occasional photophobia and effortless vomiting containing recently ingested food. She had no family history of migraine.

Fig 1: Tuberous sclerosis characteristic lesions on the face: facial angiofibromata 'adenoma sebaceum',

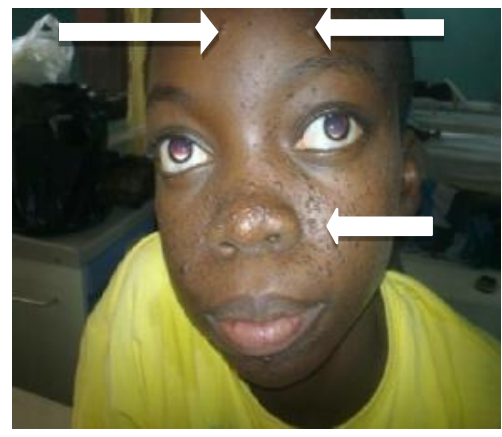

Fig 2A: Roughened, raised lesion with an orange peel consistency on the left hypochondrial region:The Shagreen patches.
Deterioration in academic performance accompanied a progressive loss of vision in both eyes. No personality change or excessive weight changes was noticed. She had an episode of generalized tonic convulsion that lasted about 20 minutes with no loss of sphincter control four days prior to presentation.

The growth and development were normal, she was fully immunized according to NPI schedule. She is the first of the four children in a monogamous setting.

She had a weight of $37 \mathrm{~kg}$ and occipito-frontal diameter of $57 \mathrm{~cm}$. No lymph node enlargement, digital clubbing, or abnormal nail changes on examination of the centre nervous system. She was well oriented in place, time and person. Short and long term memory was intact. No cranial nerve palsy; power, tone and reflexes were normal. No evidence of cerebellar lesions.

Ophthalmic evaluation showed a visual acuity of being able to count fingers at not more than one meter from the face and only perception of light in the right and left eye respectively, both eyes had brisk pupillary activities, good mydriasis and clear media. The retinal and optic nerve head appeared normal in the right eye whereas in the left eye was a huge tuberous hamartoma of the optic disc and macular as well as generalised vascular occlusion and sub-retinal fluid.

Prior to presentation at our facility she had been managed as a case of typhoid at various private health facilities for about one year when she presented with the same clinical presentation.

\section{Investigations}

Serum electrolytes, urea and creatinine were essentially normal, the ECG showed sinus tachycardia with left ventricular hypertrophy. Abdomino-pelvic ultrasonography revealed bilateral kidney enlargement with multiple hyper-echoic foci and evidence of increased vascularity

Computerized tomography (CT) scan of the brain (Fig 3 \& 4) showed hyper dense lesion on left orbit, a huge supratentorial mass crossing the midline, projecting into the lateral ventricle with associated dilatation. Also, there were intraventricular tumours arising from the 3rd ventricle extending to the anterior horn and body of the lateral ventricle. The CT scan also revealed calcification within tumours and subependymal area with associated obstructive hydrocephalus. Repeat CT scan 7 months after a Ventriculo-Peritoneal (VP) shunt showed dilated lateral ventricles with massive enlargement of the subependymal tumours projecting into the lateral ventricles

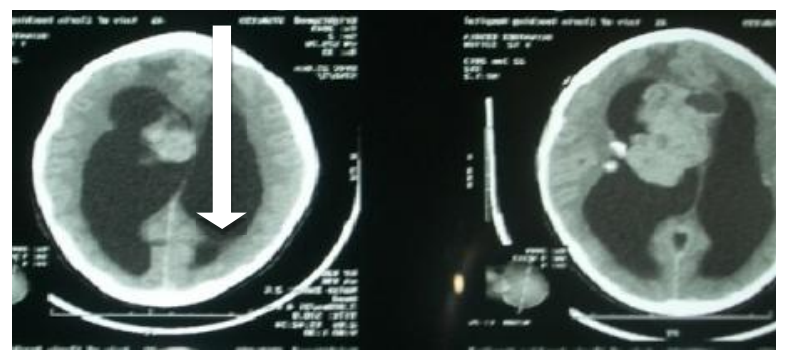


Fig 4: Repeat CT scan showing huge tumour with dilatation of the ventricles secondary to obstruction of CSF drainage

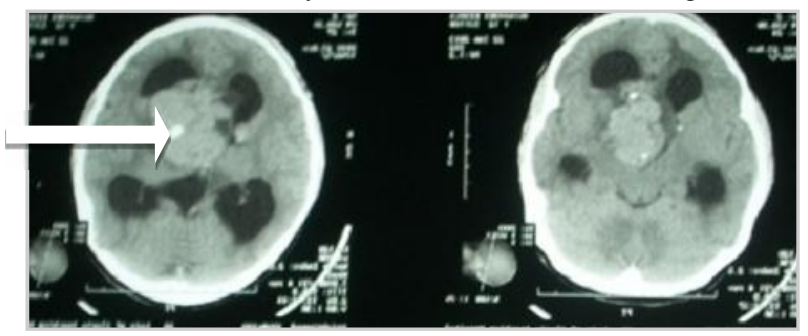

Fig 5: Brain CT scan showing calcified lesion on the retina
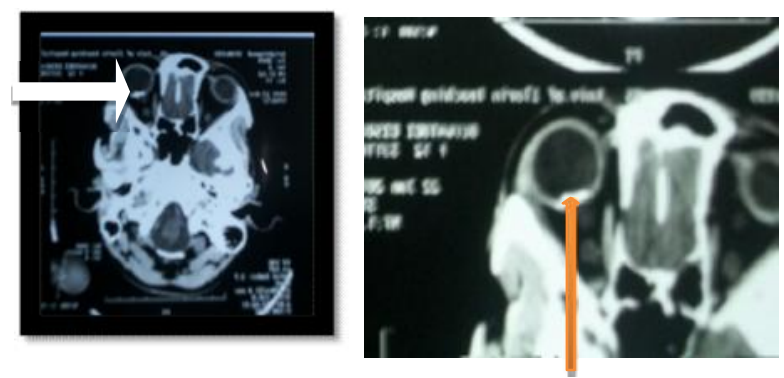

Management of the patient was by a multidisciplinary team involving the paediatrician, neurosurgeon, ophthalmologist and the radiologist. A case review and management of the patient involving all the above specialists was conducted and a joint decision on the definitive management taken. On the 16th day of admission patient had bilateral ventriculo-peritoneal shunt (V-P shunt) done which was well tolerated. The option of tumour removal cannot be done in our facility. Headache subsided and vision improved. The V-P shunt helped to decompress the brain and reduced the tension created by the accumulated CSF. Patient was discharged home for follow up.

Seven month after the V-P shunt, her condition deteriorated with evidence of raised intracranial pressure from which she died.

\section{Discussion \\ Blindness as a manifestation of TSC is not common. There was no blindness in a population based review of 100 cases by Rowley ${ }^{4}$. Delay in diagnosis is not unusual in our environment, this reflects the general health situa- tion and health seeking behaviour in our society. \\ In this case, an established school health and in particu- lar, school eye health services would have identified the child early enough for a better outcome.}

The blindness in this case is believed to have resulted primarily from the intra-cranial manifestation of the disease with tuberous masses along the visual pathway and secondary raised intracranial pressure, retinal and optic nerve harmatomas.

There is paucity of published reports on TSC with blindness in the sub-region. Most of the reported cases of ophthalmic manifestation of TSC are from Europe and America $^{4,5}$. This is not unconnected with the prohibitive cost of diagnostic investigations like CT scan or
MRI in the sub-region ${ }^{7}$. Our patient presented with deteriorating vision and deteriorating academic performance. Tumour involving the optic nerve and the retina constitute the major ophthalmic manifestation of TSC. Retinal and nonretinal findings have been documented in $\mathrm{TSC}^{4,5}$. The nonretinal finding include eyelid angiofibromas, strabismus, cataracts, colobomas, and iris depigmentation $^{8}$.

Tuberous sclerosis is inherited as an autosomal dominant inheritance with a variable penetrance. Our patient demonstrated this familial inheritance with two other family members, the mother and one of the siblings, presenting with classical facial manifestations of adenoma sebaceous. Variable expressivity is a recognised characteristic of Tuberous sclerosis ${ }^{6,7}$. TSC is a neurocutaneous disorder that is associated with tumours in various body organs especially the brain and the kidney ${ }^{8}$. The clinical manifestation of TSC varies with age of patients and also on the affected organs. Our patient presented with recurrent headache, convulsion, deteriorating vision with eventual blindness, and decline in academic performance. These are manifestations of cortical tumours. CNS tumours are found in $5 \%$ to $15 \%$ of patients with TSC. These tumours differ in their location, radiological characteristics, and biological behaviour ${ }^{9}$ Subependymal nodules are present in almost $80 \%$ of patients and can be identified prenatally or at birth. They are commonly low-grade dysplasia, which share histology with subependymal giant cell astrocytoma, whose presentation may vary from totally asymptomatic to obstructive hydrocephalus and death.

There are diagnostic criteria that have been approved for the diagnosis of TSC (Table 1). The diagnostic criteria are the outcome of a consensus conference organised by the National Institute of Health in 1998 to standardize the diagnostic criteria for $\mathrm{TSC}^{3}$. Clinical manifestations and radiologic features are used in this diagnostic criteria. A diagnosis of TS is made if a patient has two of the major criteria or one major or two minor criteria (Table 1).

Table 1: Diagnostic Criteria for Tuberous sclerosis(3)

\begin{tabular}{|c|c|}
\hline Major feature & Minor feature \\
\hline Facial angiofibromas & Multiple pits in dental enamel \\
\hline Ungula or peri-ungual fibroma & Hamartomatous rectal polyps \\
\hline Shagreen patch & Bone cysts \\
\hline Cortical tuber & Gingival fibroma \\
\hline Subependymal nodule & Nonrenal hamartoma \\
\hline $\begin{array}{l}\text { Subependymal giant cell astro- } \\
\text { cytoma }\end{array}$ & Retinal achromic patch \\
\hline $\begin{array}{l}\text { Multiple retinal nodular hamar- } \\
\text { toma }\end{array}$ & "confetti" skin lesion \\
\hline $\begin{array}{l}\text { Cardiac rhabdomyoma } \\
\text { Renal angiomyolypoma }\end{array}$ & Multiple renal cysts \\
\hline
\end{tabular}

Definite TSC - Either two major features or one major feature plus two minor features

Probable TSC - One major plus one minor feature

Possible TSC - Either one major feature or two or more minor feature

In a resource poor environment where healthcare financing is mainly out of pocket there is a great challenge to 
the number of diagnostic investigations that can be done. The diagnostic criteria are of great importance in this situation. Our patient has more than three major features of this diagnostic criteria (Shagreen patches, facial angiofibromas, cortical tubers, subependymal astrocytoma) We were able to do a brain CT scan which showed evidence of massive tumours obstructing the foramen and also compressing on the optic disc bilaterally.

\section{Conclusion}

The diagnosis of tuberous sclerosis complex was based on the lesions found on clinical examination, imaging, and pathologic studies. The blindness was multifactorial in cause including intracranial, retinal and optic nerve tumours. Comprehensive medical history, detailed physical examinations and neuroimaging study are essential in making a diagnosis of TSC. Our patient was mis-diagnosed at various health facilities for many years. This delay in making appropriate diagnosis and instituting treatment could have contributed to the eventual outcome.

Conflict of Interest: None

Funding: None

\section{References}

1. Barron R, Kainulainen V, Forrest C, Krafchik B, Mock D, Sandor G. Tuberous sclerosis: clinicopathologic features and review of the literature. J Cranio-maxillofacial surgery. 2002;30(6):361-6.

2. Kliegman R, Behrman RE, Jenson HB, Stanton BF. Nelson textbook of Pediatrics: Elsevier/Saunders; 2012.

3. Hyman MH, VH W. National Institutes of Health consensus conference: tuberous sclerosis complex. Arch Neurol. 2000;57:662-5.
4. Rowley S, O'callaghan F, Osborne J. Ophthalmic manifestations of tuberous sclerosis: a population based study. British J Ophthalmology. 2001;85(4):420-3.

5. Robertson DM. Ophthalmic manifestations of tuberous sclerosis. Annals of the New York Academy of Sciences. 1991;615(1):17-25.

6. NC N, WG. P. Diagnostic and genetical aspects of tuberous sclerosis. J Med Genet 1968;5:273-80.
7. Bundey S, J EK, . Tuberous sclerosis: a genetic study. Neurol Neurosurg Psychiatry. 1969;32:591-603.

8. Millar S, Bradley L, Donnelly DE, et al. Familial pediatric endocrine tumors. Oncologist 2011;16:1388-96.

9. Torres OA, Roach E, Delgado MR, Sparagana SP, Sheffield E, Swift $\mathrm{D}$, et al. Early diagnosis of subependymal giant cell astrocytoma in patients with tuberous sclerosis. J Child Neurology. 1998;13(4):173-7. 